\title{
Mobile High Resolution Xenon Nuclear Magnetic Resonance Spectroscopy in the Earth's Magnetic Field
}

\author{
Stephan Appelt, ${ }^{1, *}$ F. Wolfgang Häsing, ${ }^{1}$ Holger Kühn, ${ }^{2}$ Juan Perlo, ${ }^{2}$ and Bernhard Blümich ${ }^{2}$ \\ ${ }^{1}$ Zentralinstitut für Elektronik, Forschungszentrum Jülich, D-52425 Jülich, Germany \\ ${ }^{2}$ Institut für Technische Chemie und Makromolekulare Chemie, RWTH Aachen, D-52056 Aachen, Germany
} (Received 26 January 2005; published 17 May 2005)

\begin{abstract}
Conventional high resolution nuclear magnetic resonance (NMR) spectra are usually measured in homogeneous, high magnetic fields $(>1 \mathrm{~T})$, which are produced by expensive and immobile superconducting magnets. We show that chemically resolved xenon (Xe) NMR spectroscopy of liquid samples can be measured in the Earth's magnetic field $\left(\sim 5 \times 10^{-5} \mathrm{~T}\right)$ with a continuous flow of hyperpolarized Xe gas. It was found that the measured normalized Xe frequency shifts are significantly modified by the Xe polarization density, which causes different dipolar magnetic fields in the liquid and in the gas phases.
\end{abstract}

The development of NMR methods in high magnetic fields has opened the door to many applications in the last decades $[1,2]$. A trend to higher magnetic fields produced by large and expensive superconducting magnets is observed because the signal-to-noise ratio $(S / N)$ and the dispersion of the different NMR lines improve with increasing field strength. Also the potential of NMR in low magnetic fields $\left(10^{-3}-1 \mathrm{~T}\right)$ is being explored, because low-field magnets are mobile and can be operated with low-cost devices [3-5]. The two crucial problems of lowfield NMR are the inherent low $S / N$ and the low spectral resolution due to the fact that the spectral lines are broad compared to the line separation. Recently it was demonstrated that chemical shift resolved NMR spectra can be obtained for protons $\left({ }^{1} \mathrm{H}\right)$ in an inhomogeneous high field by using an ingenious radio-frequency pulse excitation [5] and for ${ }^{129} \mathrm{Xe}$ in organic liquids and in polypropylene in magnetic fields as low as $25 \mathrm{G}[6-8] .{ }^{1} \mathrm{H}$ NMR was conducted in the Earth's magnetic field $[9,10]$ of Antarctica on half-liter size ice samples $[11,12]$ and other places on ground water $[13,14]$ with sample volumes larger than $1 \mathrm{~m}^{3} . J$ couplings were measured by ultralow field SQUID detected NMR of ${ }^{1} \mathrm{H}$ and of ${ }^{31} \mathrm{P}$ in micro-Tesla fields [15]. The development of hyperpolarization technology [16-20] stimulates new applications of low-field NMR. The main advantage of hyperpolarized nuclei like ${ }^{129} \mathrm{Xe}$ is the large nuclear spin polarization, independent of the magnetic field strength $B_{0}$. Consequently, NMR [6-8] and magnetic resonance imaging [21-23] with hyperpolarized nuclei can be performed in very low magnetic fields.

In this Letter we demonstrate for the first time that high resolution Xe NMR can be performed in the Earth's magnetic field, allowing a direct observation of the large chemical shift differences of ${ }^{129} \mathrm{Xe}$ gas and Xe dissolved in various organic liquids, and of the dipolar magnetic field generated by the hyperpolarized ${ }^{129} \mathrm{Xe}$.

Assuming a nuclear induction NMR spectrometer in the Earth's field, where the only noise source is the
Johnson noise of the coil $N_{J}=\sqrt{4 k T R_{w} \Delta \nu_{b}}(T=$ absolute temperature, $\quad R_{w}=$ coil resistance, $\quad \Delta \nu_{b}=$ detection bandwidth), the $S / N$ for $1 \mathrm{~cm}^{3}$ of water in a single-scan experiment is about $10^{-2}[1,24]$. For a $S / N \sim$ 10 at least $10^{6}$ averages are necessary, and therefore thermally polarized Earth's field proton NMR for small sample volumes is not feasible. In order to measure an Earth's field Xe NMR signal of $\mathrm{a} \mathrm{cm}^{3}$ sized sample with a single scan, the Xe nuclear spins have to be hyperpolarized. Defining $P_{\mathrm{Xe}}$ as the degree of hyperpolarization, the limiting $S / N$ is given by

$$
\frac{S}{N}=\frac{\frac{3}{4} \gamma_{\mathrm{Xe}} n_{\mathrm{Xe}} \frac{B_{1}}{i} \hbar \omega_{0} P_{\mathrm{Xe}}}{2 \sqrt{2} N_{J}},
$$

where $n_{\mathrm{Xe}}$ is the number of ${ }^{129} \mathrm{Xe}$ atoms, $\omega_{0} \sim 2 \pi \times$ $570 \mathrm{~Hz}$ is the ${ }^{129} \mathrm{Xe}$ Larmor frequency in the Earth's field, and $\gamma_{\mathrm{Xe}}=2 \pi \times 1178 \mathrm{~s}^{-1} \mathrm{G}^{-1}$ is the Xe gyromagnetic ratio. From Eq. (1) it follows that the $S / N$ for $1 \mathrm{~cm}^{3}$ of hyperpolarized Xe gas at 1 bar pressure $\left(P_{\mathrm{Xe}}=0.3, n_{\mathrm{Xe}}=\right.$ $\left.7.5 \times 10^{18}\right)$ is about 30 if we assume for the coil sensitivity $B_{1} / i=70 \mathrm{GA}^{-1}$ and $N_{J} \sim 5 \mathrm{nV}$.

The second crucial problem for Earth's field NMR is the spectral resolution. A high resolution NMR spectrum of a nuclear species is obtained when most of the NMR lines originating from chemically nonequivalent sites are well resolved. For protons in the Earth's field, high resolution NMR spectroscopy is not possible because the frequency separation of the nonequivalent protons (about $10^{-2} \mathrm{~Hz}$ ) is much smaller than the typical proton linewidths $w_{\mathrm{H}}$ of about $1 \mathrm{~Hz}$. Fortunately for ${ }^{129} \mathrm{Xe}$ the situation is quite different because the transverse $T_{2}$ relaxation times of ${ }^{129} \mathrm{Xe}$ in liquid samples vary between $10-1000 \mathrm{~s}$ [6], and the chemical shift for liquids ranges between 100 and $300 \mathrm{ppm}$. For ${ }^{129} \mathrm{Xe}$ dissolved in a pure organic solvent $\left(T_{2}{ }^{\mathrm{Xe}} \sim 100 \mathrm{~s}, w_{\mathrm{Xe}} \sim 6 \times 10^{-3} \mathrm{~Hz}\right.$ FWHM $)$ and for a chemical shift difference between $\mathrm{Xe}$ gas and $\mathrm{Xe}$ in the solvent of $\delta_{\mathrm{Xe}} \sim 200 \mathrm{ppm}$, we can estimate the lowest field $B_{\min }$ for which the two NMR lines (Xe gas and Xe in 
solution) are separated as

$$
B_{\min }=\frac{2 \pi w_{\mathrm{Xe}}}{\gamma_{\mathrm{Xe}} \delta_{\mathrm{Xe}}}=2.8 \times 10^{-6} \mathrm{~T} .
$$

Because $B_{\min } \ll 5 \times 10^{-5} \mathrm{~T}$, the chemical shift can be well resolved in the Earth's field by Xe NMR spectroscopy.

In order to investigate the potential of Xe NMR in the Earth's field we built a low frequency NMR spectrometer and a mobile $\mathrm{Rb}$-Xe hyperpolarizer (Fig. 1) based on $\mathrm{Rb}-\mathrm{Xe}$ spin-exchange optical pumping [18-20]. The hyperpolarizer produces a continuous flow of $\sim 2.5 \times 10^{19}$ ${ }^{129} \mathrm{Xe}$ atoms per minute with a polarization fraction of $P_{\mathrm{Xe}}=0.3$. The polarized Xe gas is transferred via a $7 \mathrm{~m}$ long plastic tube to a liquid sample $\left(2 \mathrm{~cm}^{3}\right)$ into the NMR probe. The continuous flow guarantees a permanent exchange of hyperpolarized $\mathrm{Xe}$ atoms between the gas phase and the liquid phase. The ${ }^{129} \mathrm{Xe}$ nuclei in the Earth's field are excited by a $90^{\circ} \mathrm{dc}$ magnetic field pulse (saddle coil, $B_{1}=1.8 \mathrm{G}, 120 \mu \mathrm{s}$ duration). The following freeinduction decay (FID) is picked up by a NMR coil tuned to $570 \mathrm{~Hz}$ for ${ }^{129} \mathrm{Xe}$, amplified by a preamplifier, and processed by the NMR electronics. All measurements were performed outdoors at $10^{\circ} \mathrm{C}$ about $50 \mathrm{~m}$ away from buildings which produce ambient electromagnetic noise. This is possible due to negligible noise pickup of the small NMR coil, which is shielded by a cylinder from $20 \mathrm{~mm}$ thick aluminum.

Figure 2(a) (left) shows the measured FID of hyperpolarized $\mathrm{Xe}$ gas at 0.07 bar partial pressure which is continuously flowing over $2 \mathrm{~cm}^{3}$ of liquid toluene. A broad and a narrow line are observed in the Fourier transform (FT) spectrum [Fig. 2(a), right] corresponding to Xe gas and to $\mathrm{Xe}$ dissolved in toluene. The gas flow causes a broadening of the Xe gas peak $\left(w_{\mathrm{Xe}}=0.1 \mathrm{~Hz}\right)$ due to the excited Xe gas atoms flowing out of the sample. Figure 2(b) displays the FID of Xe in toluene after the Xe gas flow has been stopped. Obviously the beat pattern of the FID is much longer compared to that in Fig. 2(a). The solid line in Fig. 2(c) shows the FT spectrum of Fig. 2(b), where the gas peak has a width of $8 \times 10^{-3} \mathrm{~Hz}$

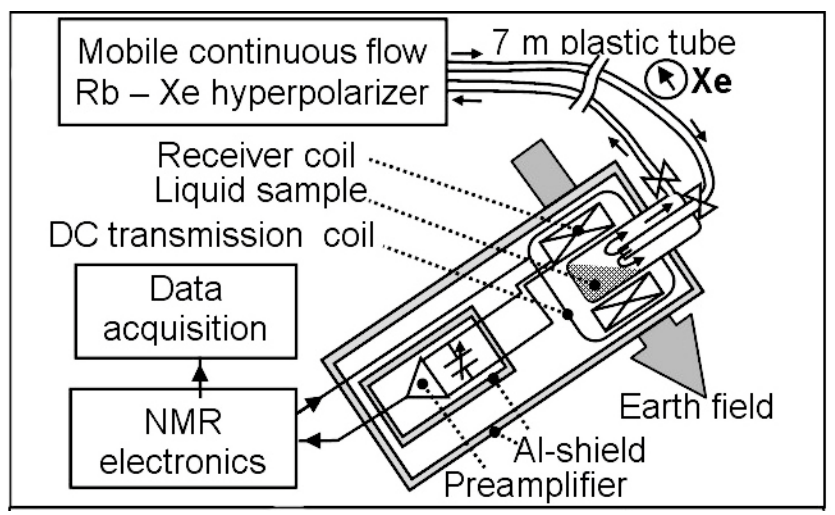

FIG. 1. Experimental setup for the Earth's field NMR.
FWHM. The gas peak is used as the reference for the zero chemical shift. The frequency difference between the two peaks $(0.1087 \mathrm{~Hz})$ divided by the Xe Larmor frequency results in a chemical shift of $191 \pm 1 \mathrm{ppm}$. The dotted line in Fig. 2(c) is the FT spectrum of Xe in ethanol with a chemical shift difference of $170 \pm 1 \mathrm{ppm}$. Both chemical shift differences are close to our values measured at $7 \mathrm{~T}$ and at $10^{\circ} \mathrm{C}$ (toluene, $192 \mathrm{ppm}$; ethanol, $169 \mathrm{ppm}$ ). We estimated the resolution for the absolute determination of the Earth's magnetic field to a few pT.

Figure 3(a) shows the frequency shift of seven different spectra of Xe in ethanol during 30 min of measuring time. The frequency shift results from fluctuations of the Earth's field $(\sim 3 \mathrm{nT})$ at this time scale. The chemical shift measurements are not influenced by these fluctuations [Fig. 3(b)] because both lines (Xe gas and Xe in ethanol) are shifted by the same value. The error for a single

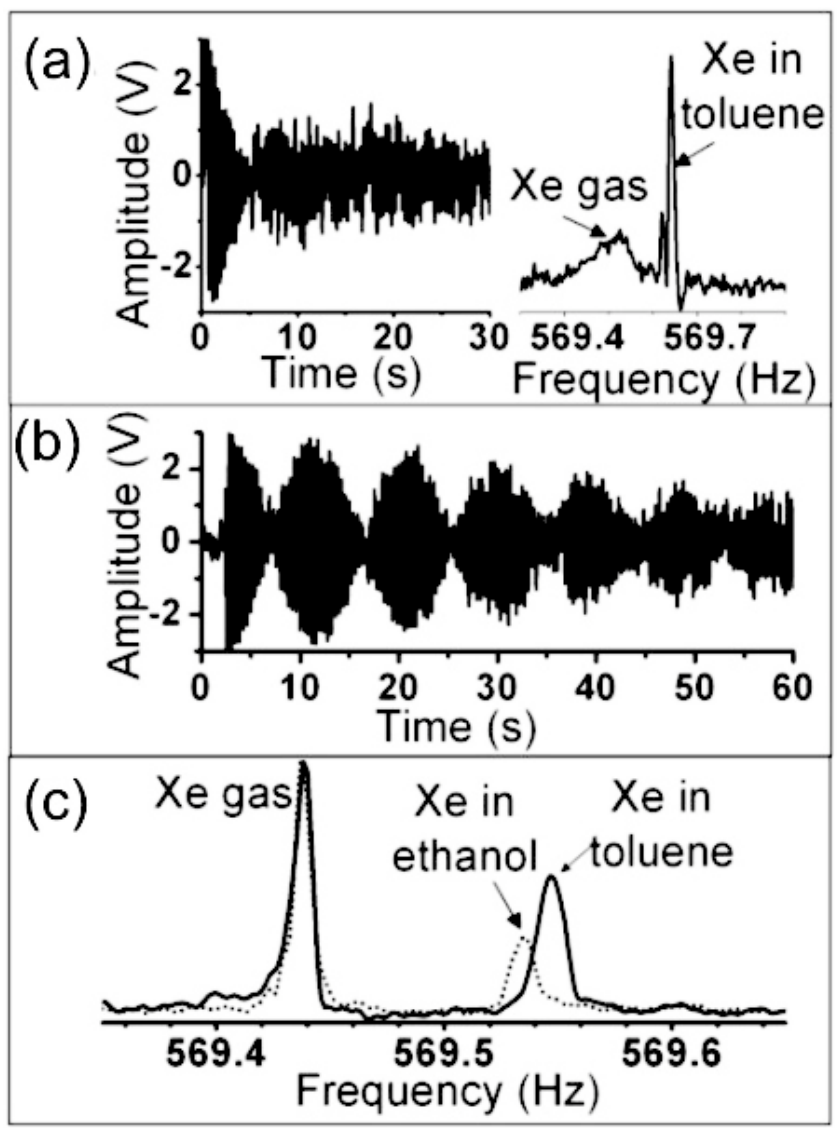

FIG. 2. Xe NMR spectroscopy in the Earth's field. (a) ${ }^{129} \mathrm{Xe}$ FID (left) and corresponding spectrum (right) measured with Xe gas flowing above $2 \mathrm{~cm}^{3}$ of liquid toluene. (b) ${ }^{129} \mathrm{Xe}$ FID as in (a) but without gas flow. Compared to (a) the transverse relaxation time of the $\mathrm{Xe}$ gas $\left(T_{2} \sim 80 \mathrm{~s}\right)$ is about 6 times longer. (c) The Fourier transform of (b) (solid line) shows two separated lines $\left(\sim 8 \times 10^{-3} \mathrm{~Hz} \mathrm{FWHM}\right)$ corresponding to $\mathrm{Xe}$ gas (left) and $\mathrm{Xe}$ in toluene (right). The dotted line displays the spectrum of $\mathrm{Xe}$ in ethanol. For all experiments the flip angle of the dc excitation pulse was $90^{\circ}$. 


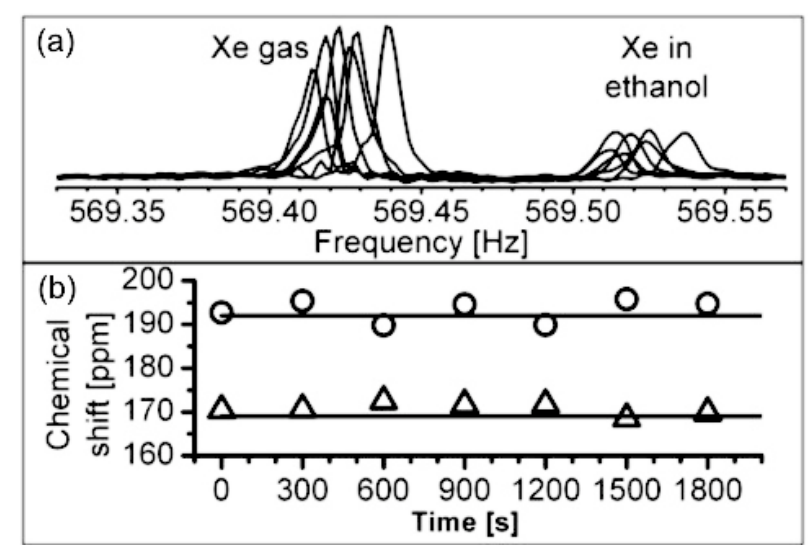

FIG. 3. Accuracy of measured Xe chemical shifts. (a) Xe spectra in ethanol measured over a time period of 30 min with $90^{\circ}$ pulses. The density of $\mathrm{Xe}$ gas ( $\mathrm{Xe}$ in ethanol) is 0.07 (0.18) amagat. (b) Xe chemical shift of toluene (circles) and of ethanol (triangles). The solid lines indicate the chemical shift measured at $7 \mathrm{~T}$.

measurement of the chemical shift is about $0.5 \mathrm{ppm}$. The scattering of a few ppm for different measurements is caused by fluctuations of the sample temperature and of the nuclear Xe polarization (see below).

Xenon earth's field NMR is very sensitive to magnetic fields produced by the sample itself [Fig. 4(a)]. At higher concentrations of hyperpolarized $\mathrm{Xe}$ in the sample the dipolar magnetic fields produced by the nuclei influence the position and the width of the Xe lines in the spectrum. Hyperpolarized Xe gas in high concentrations is produced by first accumulating the flowing gas in the form of solid $\mathrm{Xe}(T \sim 77 \mathrm{~K})$ in a field $>0.1 \mathrm{~T}$ and then thawing it to $\mathrm{Xe}$ gas. This is demonstrated in Fig. 4(b) by three different spectra of $\mathrm{Xe}$ in toluene after a $30^{\circ}$ dc pulse excitation (solid and dashed lines) and after a $90^{\circ}$ pulse excitation (dotted line). The dotted line serves as the reference spectrum at low ${ }^{129} \mathrm{Xe}$ polarization density $\sim 0.1$ amagat $\times P_{\mathrm{Xe}}$ in the liquid). The solid (dashed) line is measured at high ${ }^{129}$ Xe polarization density $\left(\sim 1\right.$ amagat $\left.\times P_{\mathrm{Xe}}\right)$ where the longitudinal Xe nuclear polarization which is left after the $30^{\circ}$ pulse excitation is pointing along (against) the direction of the Earth's field vector [Fig. 4(a)]. Compared to the position of the peak from dissolved xenon in the reference spectrum (dotted line) the maxima of the peaks of dissolved xenon at high Xe density are shifted by about $0.023 \mathrm{~Hz}$ (solid line) and by $-0.03 \mathrm{~Hz}$ (dashed line). The dipolar field of the reference spectrum is equal to zero due to the $90^{\circ}$ pulse and the low polarization density. Defining $\sigma_{\mathrm{n}}=\left(\nu_{\mathrm{lq}}^{\mathrm{Xe}}-\nu_{\mathrm{gas}}^{\mathrm{Xe}}\right) / \nu_{\mathrm{gas}}^{\mathrm{Xe}}$ as the normalized Xe frequency shift with $\nu_{\mathrm{lq}}^{\mathrm{Xe}}\left(\nu_{\mathrm{gas}}^{\mathrm{Xe}}\right)$ being the measured frequency of $\mathrm{Xe}$ in the liquid (gas) phase, the two shifts correspond to $\sigma_{\mathrm{n}}=$ $233 \mathrm{ppm}$ for the solid line and $143 \mathrm{ppm}$ for the dashed line.

Figure 4(c) shows $\sigma_{\mathrm{n}}$ versus the time after mixing of the concentrated Xe gas with the liquid for the case where the Xe polarization vector points parallel (squares) and anti-

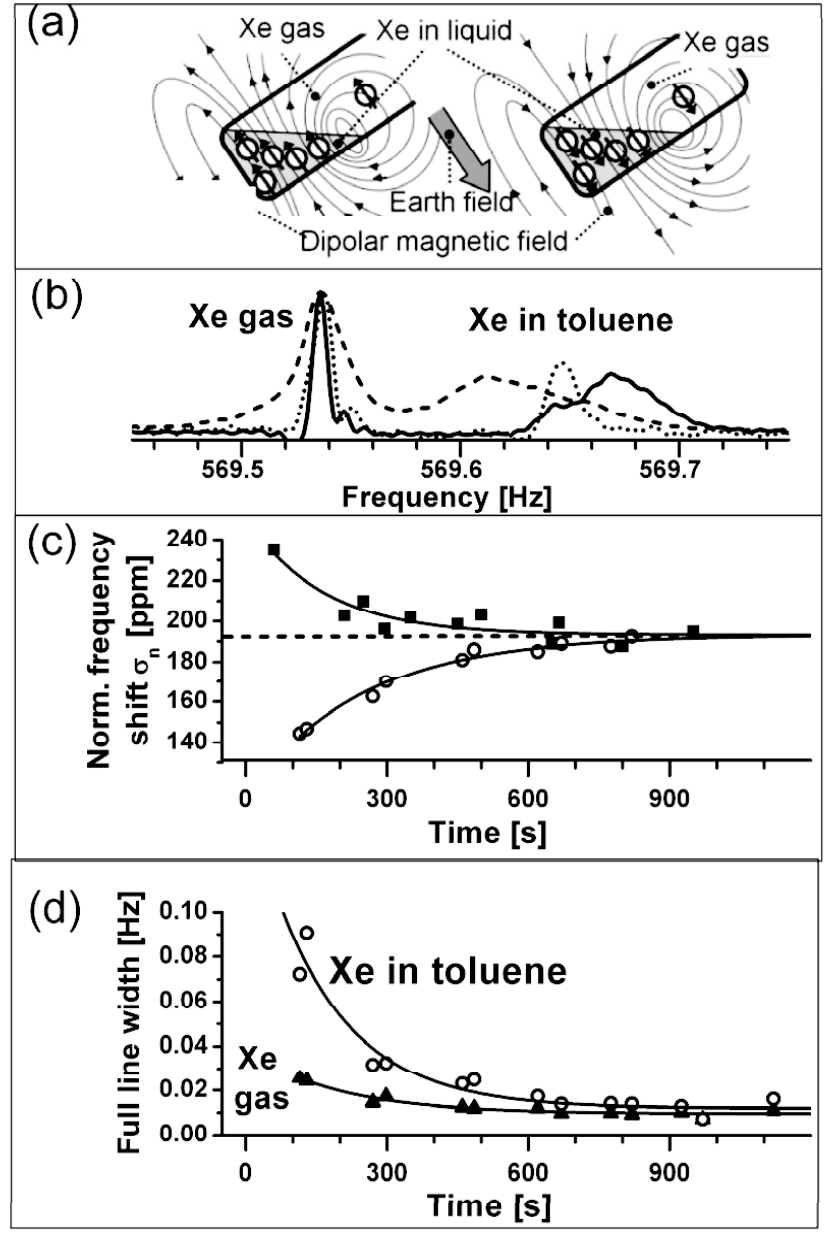

FIG. 4. Xe NMR spectroscopy at high Xe polarization densities. (a) Enhanced view of the liquid toluene sample in the Earth's magnetic field and sketch of the Xe dipolar field antiparallel and parallel to the Earth's field vector. (b) Dotted line: Reference ${ }^{129} \mathrm{Xe}$ spectrum after $90^{\circ}$ pulse with low ${ }^{129} \mathrm{Xe}$ polarization density ( 0.1 amagat $\times P_{\mathrm{Xe}}$ in the liquid). Dashed (solid) line: ${ }^{129} \mathrm{Xe}$ spectrum in the presence of high ${ }^{129} \mathrm{Xe}$ polarization density $\left(\sim 1\right.$ amagat $\times P_{\mathrm{Xe}}$ in the liquid) after a $30^{\circ}$ pulse for antiparallel (parallel) orientation of the Xe polarization vector. A positive and a negative shift as well as a broadening of the Xe peaks in toluene are observed. (c) Time dependence of the observed normalized Xe frequency shift which converges to the chemical shift of toluene $(192 \mathrm{ppm})$ for both orientations of the Xe polarization. Solid line: Exponential fit. (d) Exponential fit (solid line) of the measured linewidth of Xe gas (triangles) and $\mathrm{Xe}$ in toluene (circles).

parallel (circles) to the Earth's field vector. Both measured curves decay exponentially with the longitudinal relaxation time of $T_{1} \sim 200 \mathrm{~s}$ and converge to the Xe chemical shift value measured in high field $\left(192 \mathrm{ppm}\right.$ at $10^{\circ} \mathrm{C}$ and $7 \mathrm{~T})$. These results can be explained by different magnetic dipolar fields caused by the different nuclear polarization densities of Xe dissolved in the liquid and of the Xe gas which need to be added to the Earth's magnetic field. Because of an Ostwald solubility of five in toluene, the 
$\mathrm{Xe}$ concentration $[\mathrm{Xe}]$ in the liquid (and therefore the averaged dipolar field) is about 5 times higher than that in the gas phase. At time $t=0$ the average difference of dipolar fields between gas and liquid is $3 \mathrm{nT}$, and this average dipolar field decays with the $T_{1}$ time of $\mathrm{Xe}$ in toluene.

Numerical calculations of a homogeneously polarized liquid with a given geometry of a slanted cylinder show that the measured mean dipolar field difference of $3 \mathrm{nT}$ corresponds to a polarization density difference of $\left([\mathrm{Xe}]_{\mathrm{tol}} P_{\mathrm{Xe}}^{\text {tol }}-[\mathrm{Xe}]_{\mathrm{gas}} P_{\mathrm{Xe}}^{\mathrm{gas}}\right) \sim 1 \times 10^{18} \mathrm{~cm}^{-3}$. The calculations show also that the dipolar field inside the liquid is inhomogeneous due to the boundaries of the slanted cylinder, which leads to an additional broadening of the measured Xe spectral line in the liquid. As shown in Fig. 4(b) the two lines measured at high polarization density show a significant broadening by more than a factor of 4 compared to the width of the reference line. Because the dipolar field is proportional to the polarization density, the line broadening is expected to decay exponentially with the $T_{1}$ relaxation time. This is demonstrated in Fig. 4(d) for the two lines of Xe in toluene and for Xe gas. For Xe in toluene we observed a maximum linewidth of $0.09 \mathrm{~Hz}$ FWHM $\left(T_{2}{ }^{*} \sim 7 \mathrm{~s}\right)$ at $t=0 \mathrm{~s}$. Numerical simulations of the dipolar field distribution in the liquid predict for our experimental conditions a $T_{2}$ inh decay of about $30 \mathrm{~s}$. Further additional dephasing mechanisms occur due to dipolar interactions between the Xe atoms [25] ( $T_{2}{ }^{\text {dip }} \sim$ $25 \mathrm{~s})$ and to radiation damping [1] $\left(T_{2}{ }^{\text {rd }} \sim 20 \mathrm{~s}\right)$. The sum of all the decay rates explains our observed $T_{2}{ }^{*}$ decay of $\sim 7 \mathrm{~s}$.

In conclusion, we have measured for the first time chemical shift resolved NMR spectra of Xe in organic liquids from $\mathrm{cm}^{3}$ sized samples in the Earth's magnetic field. We found that the dipolar fields produced by the polarized Xe atoms have a substantial effect on the position and the width of the measured Xe spectral lines. Using $90^{\circ}$ pulses and low polarization densities, the accuracy of the Xe chemical shift $(<1 \mathrm{ppm})$ is comparable to that achieved by high field superconducting magnets. Many applications are envisioned, for example, the characterization of mineral oil in well logging and quality control, the measurement of the Earth's magnetic field with sub-pT resolution in geophysics, and the detection of biomagnetic fields.

The authors gratefully acknowledge generous support from $\mathrm{H}$. Halling and excellent technical assistance from $\mathrm{U}$. Sieling from Forschungszentrum Jülich.

*To whom all correspondence should be addressed. Electronic address: st.appelt@fz-juelich.de
[1] A. Abragam, The Principles of Nuclear Magnetism (Clarendon, Oxford, U.K., 1961).

[2] R. R. Ernst, G. Bodenhausen, and A. Wokaun, Principles of Nuclear Magnetic Resonance in One and Two Dimensions (Clarendon, Oxford, U.K., 1987).

[3] G. Eidmann, R. Savelsberg, P. Blümler, and B. Blümich, J. Magn. Reson., Ser. A 122, 104 (1996).

[4] F. Casanova and B. Blümich, J. Magn. Reson. 163, 38 (2003).

[5] C. A. Meriles, D. Sakellariou, D. H. Heise, A. J. Moulé, and A. Pines, Science 293, 82 (2001).

[6] S. Appelt, F. W. Häsing, S. Baer-Lang, N. J. Shah, and B. Blümich, Chem. Phys. Lett. 348, 263 (2001).

[7] S. Saxena, A. Wong-Foy, A. J. Moule, J. A. Seeley, R. McDermott, J. Clarke, and A. Pines, J. Am. Chem. Soc. 123, 8133 (2001).

[8] A. Wong-Foy, S. Saxena, A. J. Moulé, H.-M.L. Bitter, J. A. Seeley, R. McDermott, J. Clarke, and A. Pines, J. Magn. Reson. 157, 235 (2002).

[9] J. Kaplan, Phys. Rev. 93, 939 (1954); see M. Packard and R. H. Varian, p. 941.

[10] G. J. Béné, Phys. Rep. 58, 213 (1980).

[11] P. T. Callaghan, C. D. Eccles, and J. D. Seymour, Rev. Sci. Instrum. 68, 4263 (1997).

[12] P. T. Callaghan, R. Dykstra, C. D. Eccles, T. G. Haskell, and J.D. Seymour, Cold Reg. Sci. Technol. 29, 153 (1999).

[13] M. Goldman, B. Rabinovich, M. Rabinovich, D. Gilad, I. Gev, and M. Schirov, J. Appl. Geophys. 31, 27 (1994).

[14] O. A. Shushakov, Geophysics 61, 998 (1996).

[15] R. McDermott, A.H. Trabesinger, M. Mück, E. L. Hahn, A. Pines, and J. Clarke, Science 295, 2247 (2002).

[16] M. A. Bouchiat, R. R. Carver, and C. M. Varnum, Phys. Rev. Lett. 5, 373 (1960).

[17] F. D. Colegrove, L. D. Schearer, and G. K. Walters, Phys. Rev. 132, 2561 (1963).

[18] W. Happer, Rev. Mod. Phys. 44, 169 (1972).

[19] B. Driehuys, G. D. Cates, E. Miron, K. Sauer, D. K. Walter, and W. Happer, Appl. Phys. Lett. 69, 1668 (1996).

[20] S. Appelt, A. Ben-Amar Baranga, C. J. Erickson, M. V. Romalis, A. R. Young, and W. Happer, Phys. Rev. A 58, 1412 (1998).

[21] J. J. Heckman, M. P. Ledbetter, and M. V. Romalis, Phys. Rev. Lett. 91, 067601 (2003).

[22] M. Augustine, D. TonThat, A. Wong-Foy, J. L. Yarger, M. Tomaselli, J. Clarke, and A. Pines, Appl. Phys. Lett. 72, 1908 (1998).

[23] C. H. Tseng, G. P. Wong, V. R. Pomeroy, R. W. Mair, D. P. Hinton, D. Hoffmann, R. E. Stoner, F. W. Hersman, D. G. Cory, and R. L. Walsworth, Phys. Rev. Lett. 81, 3785 (1998).

[24] A. G. Webb, Prog. Nucl. Magn. Reson. Spectrosc. 31, 1 (1997).

[25] M. V. Romalis and M. P. Ledbetter, Phys. Rev. Lett. 87, 067601 (2001). 